\title{
Alpha Power Transformed Lindley Distribution
}

\author{
L A Rosa ${ }^{1 *}$, S Nurrohmah ${ }^{2}$, I Fithriani ${ }^{3}$ \\ ${ }^{1,2,3}$ Department of Mathematics, Universitas Indonesia, Depok, 16424, Indonesia \\ *) Corresponding author: lady.amanda @sci.ui.ac.id
}

\begin{abstract}
The one parameter Lindley distribustion $(\theta)$ has been widely used in various field such as biology, technique, medical, and industries. Lindley distribution is capable for modelling data with monotone increasing hazard rate. However, in real life, there are situations where the hazard rate is not monotone. Therefore, to enhance the Lindley distribution capabilities for modelling data, a modification can be used by using Alpha Power Transformed method. The result of the modification of Lindley distribution is commonly called Alpha Power Transformed Lindley distribution (APTL) distribution that has two parameters $(\alpha$, $\theta$ ). This new APTL distribution is appropriate in modelling data with decreasing or unimodal shaped of probability density function, and has hazard rates with increasing, decreasing, and upside-down bathtub shaped. The properties of the proposed distribution are discussed include probability density function, cumulative distribution function, survival function, hazard rate function, moment generating function, and $r$ th moment. The model parameters are obtained using maximum likelihood method. The waiting time data is used as an illustration to describe the utility of APTL distribution.
\end{abstract}

Keywords: hazard rate, Lindley distribution, maximum likelihood, upside-down bathtub 


\section{Introduction}

In real life, there are various kind of problems that can occur in every organism. One of the problems is the occurrence of failure. The time of an object to survive until the time of failure can be expressed as waiting time. The importance of analyzing data of waiting time for failure is the dispersion of data can be known.

One distribution that has a capability for modelling a waiting time data is Lindley distribution that was originally introduced by Dennis V. Lindley in 1958. Lindley distribution that has one parameter $(\theta)$ is formed from the mixing distribution of exponential distribution $(\theta)$ and gamma distribution $(2, \theta)$. Ghitany et al. provided a comparison of statistical properties from Lindley distribution and exponential distribution, it shows that Lindley distribution performs better than exponential distribution in modelling a waiting time data. It is because exponential distribution has a constant hazard rate. Essentially, it is very rare to find waiting time data that have a characteristics of a constant hazard rates, where it indicates that the object will have the same risk of failure every time. The Lindley distribution has characteristics related to a monotone increasing hazard rate, therefore Lindley distribution more flexible than exponential distribution.

Lindley distribution is capable for modelling waiting time data with monotone increasing hazard rate. However, there are situations where the hazard rate is monotone decreasing or not monotone. For example, Kus C. found that the occurrence of earthquakes in the last century in North Anatolia fault zone follows decreasing hazard rate. Efron et al. analyzed the hazard rate of data set in the context head and neck cancer in Northern California Oncology Group shows upside-down bathtub shapes where the hazard rate initially increased, attained a maximum and decreasing after the patients stabilized owing a therapy. To enhance Lindley distribution capabilities for modelling various data, a modification needs to be done that retains the original 
characteristics of Lindley distribution, so that it can be used to modelling waiting time data with a non-monotone hazard rate.

The modification to increases the flexibility of Lindley Distribution can use Alpha Power transformation method as suggested by Mahdavi and Kundu in 2017. It works by adding a new parameter shape $\alpha$ to the distribution for increasing the flexibility in modelling data that has diverse characteristics in its hazard rate. The results of modification to Lindley distribution using the Alpha Power transformation called Alpha Power Transformed Lindley (APTL) distribution which has two parameters $\alpha$ and $\theta$, and will also have monotone increasing, monotone decreasing, and upside-down bathtub hazard rate.

This paper is organized as follows: the Lindley distribution, theoretical basis and formulation of Alpha power transformation is presented in section 2. Mathematical properties of APTL distribution and the maximum likelihood estimation are presented in section 3. The analysis of real data set is presented in section 4. Finally, the conclusion is in section 5 .

\section{Methods}

\section{Lindley Distribution}

Let $X$ be random variable of Lindley distribution with parameter $\theta>0$. Recall that the pdf and cdf associated to $X$ are respectively given by

$$
f_{\theta}(x)=\stackrel{\theta^{2}}{1+\theta}(1+x) e^{-\theta x}, x>0
$$

and

$$
F_{\theta}(x)=1-\left(\frac{1+\theta+\theta x}{1+\theta}\right) e^{-\theta x}, x>0
$$

Here, we present the hazard rate function of Lindley distribution and also its 
characteristics by plots with various values of parameter. Figure 1 display a variety of possible shapes of the hazard rate Lindley distribution with the various value of the parameters.

$$
h(x)=\frac{\theta^{2}(1+x)}{1+\theta+\theta x} ; x>0
$$

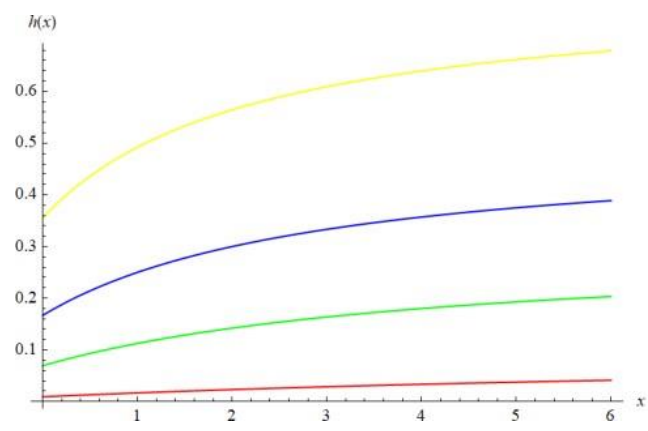

(a) Parameter $\theta<1$

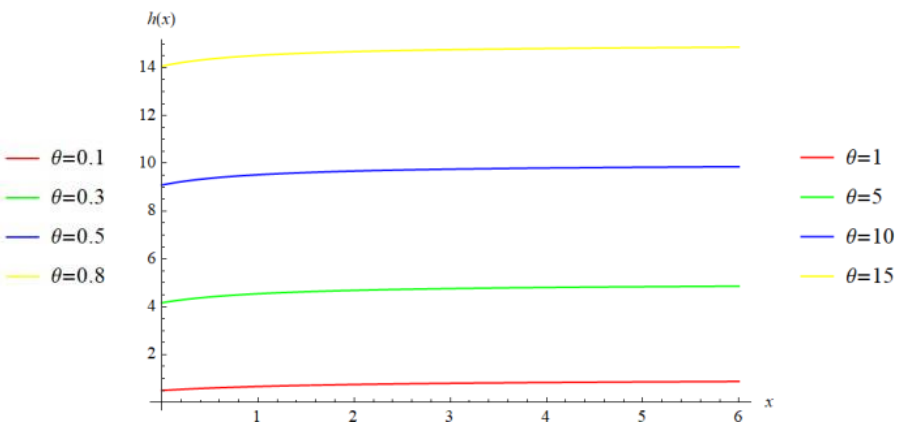

(b) Parameter $\theta \geq 1$

Figure 1. Plot of hazard rate of the Lindley distribution with various values of parameters. Figure 1 shows that Lindley distribution has only monotone increasing hazard rates.

\section{Maximum Likelihood Estimation (MLE)}

There are several methods for estimating parameter of the model under classical approach, the most commonly procedure is maximum likelihood method. In this paper, the estimation of the parameters only by maximum likelihood method. Let the random sample $X_{1}, X_{2}, \ldots, X_{n}$ with size $n$ from certain distribution with pdf $f(x ; \theta)$ that depends on $\theta \in \Omega$. Then, the joint pdf is $\left(x_{1}, x_{2}, \ldots, x_{n} ; \theta\right)=f\left(x_{1} ; \theta\right) .\left(x_{2} ; \theta\right) \ldots\left(x_{n} ; \theta\right)$. The function of joint pdf can be expressed as a function of $\theta$, can be denoted $(\theta)$ or $\left(\theta ; x_{1}, x_{2}\right.$, $\ldots, x_{n}$ ) called likelihood function, as follows;

$$
L\left(\theta ; x_{1}, x_{2}, \ldots, x_{n}\right)=f\left(x_{1} ; \theta\right) . f\left(x_{2} ; \theta\right) \ldots f\left(x_{n} ; \theta\right)=\prod_{i=1}^{n} f\left(x_{i} ; \theta\right) ; \theta \in \Omega
$$


Let $u\left(x_{1}, x_{2}, \ldots, x_{n}\right)$ be a function from $x_{1}, x_{2}, \ldots, x_{n}$ so that if $\theta$ replaced by $u\left(x_{1}, x_{2}, \ldots\right.$, $\left.x_{n}\right)$, the likelihood function $L\left(\theta ; x_{1}, x_{2}, \ldots, x_{n}\right)$ reach maximum value. So, $\left(X_{1}, X_{2}, \ldots\right.$, $X_{n}$ ) be the maximum likelihood estimator (MLE) for $\theta$ which denoted by $\hat{\theta}$ To find maximum likelihood estimator, $(\theta)$ can be modified to log-likelihood function denoted by $\ln (\theta)$. The value of $\theta$ can be find by solving first derivatives of the $\ln (\theta)$ with respect to $r, \alpha, \beta$ and equating them to zero.

\section{Alpha Power Transfromation Methods}

Alpha Power transformation method works by weighting the pdf of a distribution given by

$$
f_{A P T}(x)=\frac{w(x)}{E(w(x))} f(x), x>0
$$

Where the $(x)$ and $((x))$ is

$$
\begin{gathered}
w(x)=\alpha^{F(x)}, \alpha \neq 1 \\
E(w(x))=E\left(\alpha^{F(x)}\right)=\frac{\alpha-1}{\log \alpha}
\end{gathered}
$$

Therefore, the formulation of new pdf after transformation using Alpha Power is given by

$$
f_{A P T}(x)=\frac{\log \alpha f(x) \alpha^{F(x)}}{\alpha-1}
$$

\section{Results and Discussion}

\section{Alpha Power Transformed Lindley Distribution}

The new distribution obtained from Lindley distribution with pdf and cdf in equation (1) and (2) that has been transformed using Alpha Power transformation method in equation (5) and now let $X$ be the random variable of Alpha Power Transformed Lindley (APTL) 
distribution with parameter $\alpha>0$ and $\theta>0$. Recall that the pdf of APTL distribution is given by

$$
f_{A P T L}(x)=\left\{\begin{array}{c}
\frac{\log \alpha}{\alpha-1}\left(\frac{\theta^{2}}{1+\theta}\right)(1+x) e^{-\theta x} \alpha^{1-\left(\frac{1+\theta+\theta x}{1+\theta}\right) e^{-\theta x}} ; x>0, \alpha \neq 1 \\
\frac{\theta^{2}}{1+\theta}(1+x) e^{-\theta x} ; x>0, \alpha=1 \\
0 ; \text { elsewhere }
\end{array}\right.
$$

and characteristics pdf of APTL distribution in equation (6) will be displayed with several plots based on each parameter where the parameter conditions will be compared when $\alpha$ is constant and when $\theta$ is constant in figure (2).

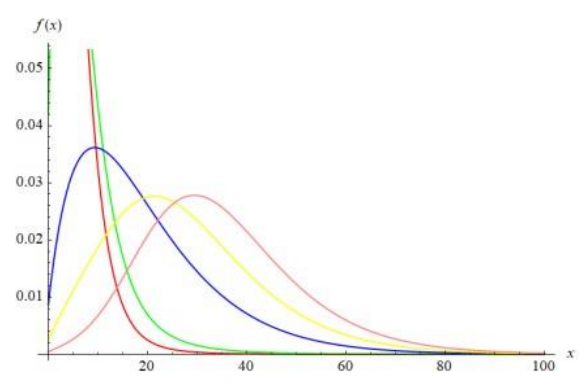

(a) Parameter $\theta$ constant $(\theta=0.1)$

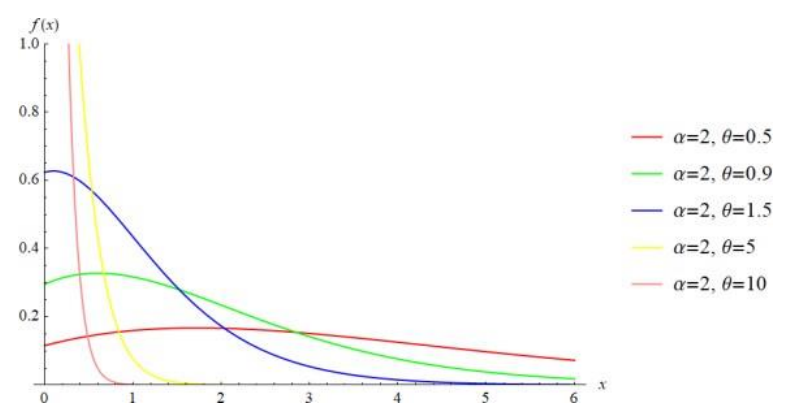

(b) Parameter $\alpha$ constant ( $\alpha=2)$

Figure 2. Plot of pdf of the APTL distribution with various values of parameters.

The plot in figure 2 shows that when parameter $\alpha$ and $\theta$ change they can produce different pdf curve that are monotone decreasing and unimodal, therefore parameter $\alpha$ and $\theta$ are parameter shape.

The corresponding cdf, survival function, and hazard rate function are respectively given by, 


$$
\begin{aligned}
& F_{A P T L}(x)=\left\{\begin{array}{c}
0 ; x \leq 0 \\
\frac{\alpha^{1-\left(\frac{1+\theta+\theta x}{1+\theta}\right) e^{-\theta x}}-1}{\alpha-1} ; x>0, \alpha \neq 1 \\
1-\left(\frac{1+\theta+\theta x}{1+\theta}\right) e^{-\theta x} ; x>0, \alpha=1
\end{array}\right. \\
& S_{A P T L}(x)=\left\{\begin{array}{c}
1 ; x \leq 0 \\
\frac{\alpha-\alpha^{1-\left(\frac{1+\theta+\theta x}{1+\theta}\right) e^{-\theta x}}}{\alpha-1} ; x>0, \alpha \neq 1 \\
\left(\frac{1+\theta+\theta x}{1+\theta}\right) e^{-\theta x} ; x>0, \alpha=1
\end{array}\right. \\
& h_{A P T L}(x)=\left\{\begin{array}{c}
0 ; x \leq 0 \\
\frac{\log \alpha\left(\frac{\theta^{2}}{1+\theta}\right)(1+x) e^{-\theta x} \alpha^{1-\left(\frac{1+\theta+\theta x}{1+\theta}\right) e^{-\theta x}}}{\alpha-\alpha^{1-\left(\frac{1+\theta+\theta x}{1+\theta}\right) e^{-\theta x}}} ; x>0, \alpha \neq 1 \\
\frac{\theta^{2}(1+x)}{1+\theta+\theta x} ; x>0, \alpha=1
\end{array}\right.
\end{aligned}
$$

and characteristics hazard rate function of APTL distribution in equation (9) will be displayed with several plots based on each parameter where the parameter conditions will be compared when $\alpha$ is constant and when $\theta$ is constant in figure 3 .

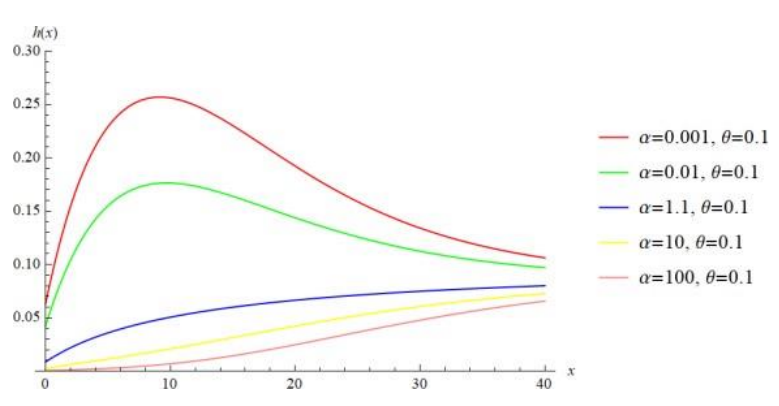

(a) Parameter $\theta$ constant $(\theta=0.1)$

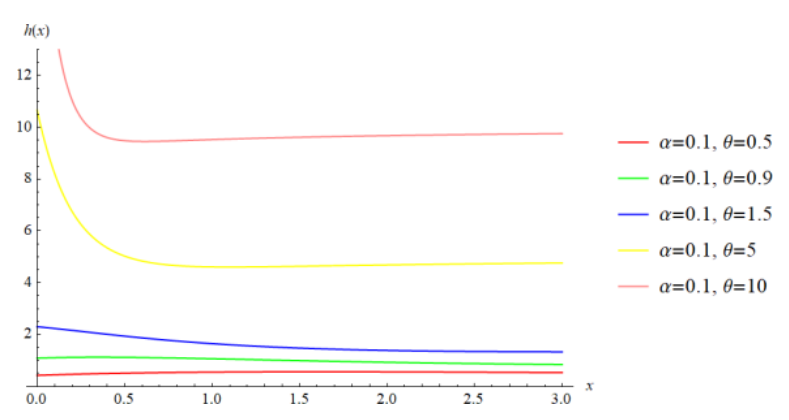

(c) Parameter $\alpha$ constant ( $\alpha=0.1)$

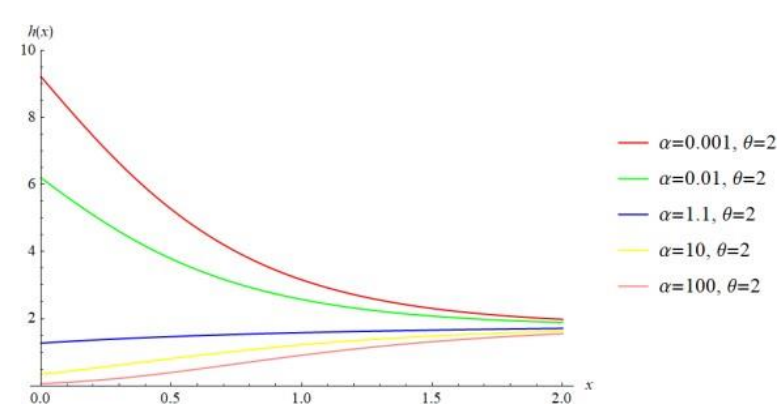

(b) Parameter $\theta$ constant $(\theta=2)$

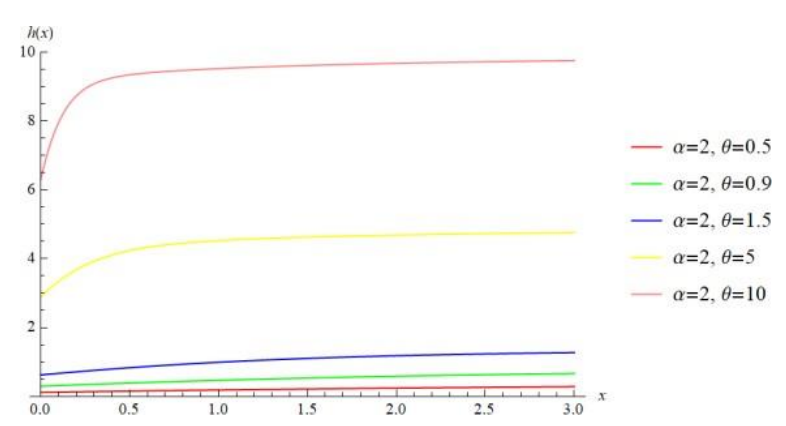

(d) Parameter $\alpha$ constant ( $\alpha=2$ ) 
Figure 3. Plot of hazard rate functions of the APTL distribution with various values of parameters.

The plot in figure 3 shows that APTL distribution have a various characteristic related to hazard rate that are monotone increasing, monotone decreasing, and upsidedown bathtub.

\section{Mathematical Properties}

Here, we present some mathematical properties such as $r$ th moment, variance, and moment generating functions of Alpha Power Transformed Lindley distribution with maximum likelihood method.

Now we present $r$ th moments and also the first moment and variance for Alpha Power Transformed Lindley (APTL) distribution. Let $X$ be random variable of APTL distribution with parameter $\alpha>0$ and $\theta>0$. Recall the pdf of APTL when $\alpha \neq 0$ in equation (5) as follows

$$
k(x)=f_{A P T L}(x ; \alpha, \theta)=\frac{\log \alpha}{\alpha-1}\left(\frac{\theta^{2}}{1+\theta}\right)(1+x) e^{-\theta x} \alpha^{1-\left(\frac{1+\theta+\theta x}{1+\theta}\right) e^{-\theta x}}
$$

Using a MacLaurin series expansion and Binomial expansion, we have $(x)$ as follows

$$
k(x)=\frac{1}{\alpha-1} \sum_{i=0}^{\infty} \sum_{j=0}^{i} \sum_{m=0}^{j} \frac{(\log \alpha)^{i+1}}{i !} \frac{\theta^{m+2}}{(1+\theta)^{m+1}}(-1)^{j}\left(\begin{array}{l}
i \\
j
\end{array}\right)\left(\begin{array}{c}
j \\
m
\end{array}\right)(1+x) e^{-\theta x(1+j)} x^{m}
$$

It follows equation (10), we obtain the $r$ th moment of the APTL distribution. The $r$ th moment is given by 


$$
\begin{aligned}
E\left(X^{r}\right)= & \int_{0}^{\infty} x^{r} k(x) d x \\
= & \int_{0}^{\infty} x^{r} \frac{1}{\alpha-1} \sum_{i=0}^{\infty} \sum_{j=0}^{i} \sum_{m=0}^{j} \frac{(\log \alpha)^{i+1}}{i !} \frac{\theta^{m+2}}{(1+\theta)^{m+1}}(-1)^{j}\left(\begin{array}{l}
i \\
j
\end{array}\right)\left(\begin{array}{l}
j \\
m
\end{array}\right)(1 \\
& +x) e^{-\theta x(1+j)} x^{m} d x \\
= & \frac{1}{\alpha-1} \sum_{i=0}^{\infty} \sum_{j=0}^{i} \sum_{m=0}^{j} \frac{(\log \alpha)^{i+1}}{i !} \frac{\theta^{m+2}}{(1+\theta)^{m+1}}(-1)^{j}\left(\begin{array}{l}
i \\
j
\end{array}\right)\left(\begin{array}{c}
j \\
m
\end{array}\right) \\
& {\left[\int_{0}^{\infty} x^{r+m} e^{-\theta x(1+j)} d x+\int_{0}^{\infty} x^{r+m+1} e^{-\theta x(1+j)} d x\right] }
\end{aligned}
$$

Using a gamma theorem, we have the $r$ th moment as follows

$$
\begin{aligned}
& E\left(X^{r}\right) \\
& =\frac{1}{\alpha-1} \sum_{i=0}^{\infty} \sum_{j=0}^{i} \sum_{m=0}^{j} \frac{(\log \alpha)^{i+1}}{i !} \frac{\theta^{m+2}}{(1+\theta)^{m+1}}(-1)^{j}\left(\begin{array}{l}
i \\
j
\end{array}\right)\left(\begin{array}{l}
j \\
m
\end{array}\right)\left[\frac{\Gamma(r+m+1)}{(\theta(1+j))^{r+m+1}}\right. \\
& \left.+\frac{\Gamma(r+m+2)}{(\theta(1+j))^{r+m+2}}\right]
\end{aligned}
$$

The result of the $r$ th moment follows by the definition of the gamma function.

From equation (11) we obtained the mean (first moment) and variance of the APTL distribution and they are respectively given by

$$
\begin{aligned}
E(X)=\frac{1}{\alpha-1} & \sum_{i=0}^{\infty} \sum_{j=0}^{i} \sum_{m=0}^{j} \frac{(\log \alpha)^{i+1}}{i !} \frac{\theta^{m+2}}{(1+\theta)^{m+1}}(-1)^{j}\left(\begin{array}{l}
i \\
j
\end{array}\right)\left(\begin{array}{c}
j \\
m
\end{array}\right)\left[\frac{\Gamma(m+2)}{(\theta(1+j))^{m+2}}\right. \\
& \left.+\frac{\Gamma(m+3)}{(\theta(1+j))^{m+3}}\right]
\end{aligned}
$$

and

$$
\begin{aligned}
& \sigma^{2}=\operatorname{Var}(X)=\left[\frac { 1 } { \alpha - 1 } \sum _ { i = 0 } ^ { \infty } \sum _ { j = 0 } ^ { i } \sum _ { m = 0 } ^ { j } \frac { ( \operatorname { l o g } \alpha ) ^ { i + 1 } } { i ! } \frac { \theta ^ { m + 2 } } { ( 1 + \theta ) ^ { m + 1 } } ( - 1 ) ^ { j } ( \begin{array} { c } 
{ i } \\
{ j }
\end{array} ) ( \begin{array} { c } 
{ j } \\
{ m }
\end{array} ) \left[\frac{\Gamma(m+3)}{(\theta(1+j))^{m+3}}+\right.\right. \\
& \left.\left.\frac{\Gamma(m+4)}{(\theta(1+j))^{m+4}}\right]\right]-\left\{\frac { 1 } { \alpha - 1 } \sum _ { i = 0 } ^ { \infty } \sum _ { j = 0 } ^ { i } \sum _ { m = 0 } ^ { j } \frac { ( \operatorname { l o g } \alpha ) ^ { i + 1 } } { i ! } \frac { \theta ^ { m + 2 } } { ( 1 + \theta ) ^ { m + 1 } } ( - 1 ) ^ { j } ( \begin{array} { c } 
{ i } \\
{ j }
\end{array} ) ( \begin{array} { c } 
{ j } \\
{ m }
\end{array} ) \left[\frac{\Gamma(m+2)}{(\theta(1+j))^{m+2}}+\right.\right. \\
& \left.\left.\frac{\Gamma(m+3)}{(\theta(1+j))^{m+3}}\right]\right\}^{2}
\end{aligned}
$$

Let $X$ be random variable of APTL distribution with parameter $\alpha>0$ and $\theta>0$.

Recall the pdf of APTL when $\alpha \neq 0$ in equation (9), therefore the mgf of APTL

distribution as follows 


$$
\begin{aligned}
M_{X}(t)= & E\left(e^{t x}\right)=\int_{0}^{\infty} e^{t x} k(x) d x \\
= & \int_{0}^{\infty} e^{t x} \frac{1}{\alpha-1} \sum_{i=0}^{\infty} \sum_{j=0}^{i} \sum_{m=0}^{j} \frac{(\log \alpha)^{i+1}}{i !} \frac{\theta^{m+2}}{(1+\theta)^{m+1}}(-1)^{j}\left(\begin{array}{l}
i \\
j
\end{array}\right)\left(\begin{array}{c}
j \\
m
\end{array}\right)(1+x) e^{-\theta x(1+j)} x^{m} \\
= & \frac{1}{\alpha-1} \sum_{i=0}^{\infty} \sum_{j=0}^{i} \sum_{m=0}^{j} \frac{(\log \alpha)^{i+1}}{i !} \frac{\theta^{m+2}}{(1+\theta)^{m+1}}(-1)^{j}\left(\begin{array}{l}
i \\
j
\end{array}\right)\left(\begin{array}{c}
j \\
m
\end{array}\right) \int_{0}^{\infty} x^{m} e^{-x[\theta(1+j)-t]} d x \\
& +\int_{0}^{\infty} x^{m+1} e^{-x[\theta(1+j)-t]} d x
\end{aligned}
$$

Using a gamma theorem, we have the mgf of APTL distribution as follows

$$
\begin{gathered}
E\left(e^{t X}\right)=\frac{1}{\alpha-1} \sum_{i=0}^{\infty} \sum_{j=0}^{i} \sum_{m=0}^{j} \frac{(\log \alpha)^{i+1}}{i !} \frac{\theta^{m+2}}{(1+\theta)^{m+1}}(-1)^{j}\left(\begin{array}{l}
i \\
j
\end{array}\right)\left(\begin{array}{c}
j \\
m
\end{array}\right)\left[\frac{\Gamma(m+1)}{(\theta(1+j)-t)^{m+1}}+\right. \\
\left.\frac{\Gamma(m+2)}{(\theta(1+j)-t)^{m+2}}\right]
\end{gathered}
$$

\section{Parameter Estimation of APTL Distribution}

Let $X_{1}, X_{2}, \ldots, X_{n}$ be a random sample of size $n$ from APTL distribution with parameter $\alpha$ and $\theta$ and the pdf is given in equation (5), the associated log-likelihood function will be

$$
\begin{gathered}
\ln L(\alpha, \theta ; x)=n\left(\ln \left(\frac{\log \alpha}{\alpha-1}\right)\right)+n(2 \ln \theta-\ln (1+\theta))+\sum_{i=1}^{n} \ln \left(1+x_{i}\right)-\theta \sum_{i=1}^{n} x_{i}+ \\
\ln \alpha \sum_{i=1}^{n}\left(1-\left(\frac{1+\theta+\theta x_{1}}{1+\theta}\right) e^{-\theta x_{1}}\right)
\end{gathered}
$$

Therefore, the maximum likelihood equations to find the parameter estimation of $\alpha$ and $\theta$ are respectively given by

and

$$
\frac{\partial \ln L(\alpha, \theta ; x)}{\partial \alpha}=n\left(\frac{1}{\alpha \log \alpha}-\frac{1}{\alpha-1}\right)+\frac{1}{\alpha} \sum_{i=1}^{n}\left(1-\left(\frac{1+\theta+\theta x_{i}}{1+\theta}\right) e^{-\theta x_{i}}\right)
$$

$$
\frac{\partial \ln L(\alpha, \theta ; x)}{\partial \theta}=n\left(\frac{2}{\theta}-\frac{1}{1+\theta}\right)-\sum_{i=1}^{n} x_{i}+\log \alpha \sum_{i=1}^{n}\left(\frac{\theta}{1+\theta}\right) e^{-\theta x_{i}}\left(1+x_{i}\right)
$$

To obtain the maximum likelihood estimates of $\alpha$ and $\theta$, we can equating the equation (12) and (13) to zero and solving simultaneously, but because the functions of maximum 
likelihood are not linear, the numerical iterative techniques must be used to solve the equations with the analytical software such as Rstudio and Mathematica.

\section{Application}

In this section, real data set will be fit to the Lindley distribution and APTL distribution to compare which distribution fits well to the data set. The data set are given by Sanku Dey et al. (2017) and represents the waiting time (in days) until 72 guinea pigs infected with virulent tubercle bacilli are dead:

Table 1. Data set of waiting time (in days) of infected guinea pigs

\begin{tabular}{|c|c|c|c|c|c|c|c|c|}
\hline 0.1 & 0.33 & 0.44 & 0.56 & 0.59 & 0.72 & 0.74 & 0.77 & 0.92 \\
\hline 0.93 & 0.96 & 1 & 1 & 1.02 & 1.05 & 1.07 & 1.07 & 1.08 \\
\hline 1.08 & 1.08 & 1.09 & 1.12 & 1.13 & 1.15 & 1.16 & 1.2 & 1.21 \\
\hline 1.22 & 1.22 & 1.24 & 1.3 & 1.34 & 1.35 & 1.39 & 1.44 & 1.46 \\
\hline 1.53 & 1.59 & 1.6 & 1.63 & 1.63 & 1.68 & 1.71 & 1.72 & 1.76 \\
\hline 1.83 & 1.95 & 1.96 & 1.97 & 2.02 & 2.13 & 2.15 & 2.16 & 2.22 \\
\hline 2.3 & 2.31 & 2.4 & 2.45 & 2.51 & 2.53 & 2.54 & 2.54 & 2.78 \\
\hline 2.93 & 3.27 & 3.42 & 3.47 & 3.61 & 4.02 & 4.32 & 4.58 & 5.55 \\
\hline
\end{tabular}

We compare the fits of the APTL distribution with the Lindley distribution. The estimation of the model parameters obtained by using software Rstudio with the maximum likelihood method.

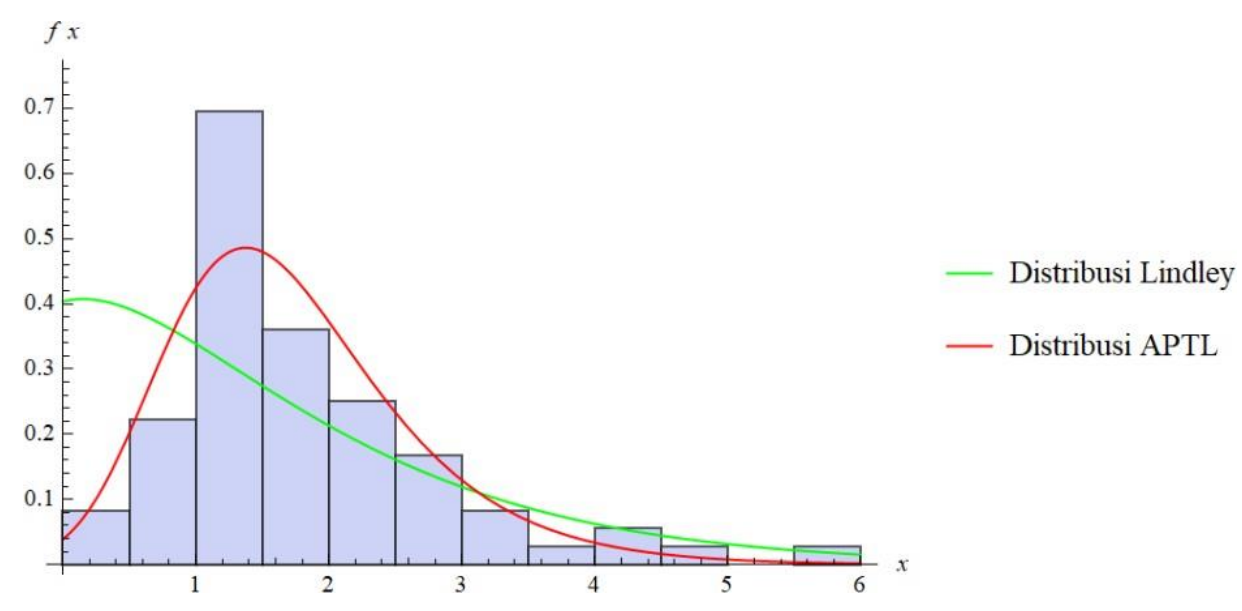

Figure 3. Histogram of the data set, plot of pdf for Lindley distribution and 
APTL distribution.

Table 2. Estimates of the parameters and Kolmogorov-Smirnov statistics

\begin{tabular}{|c|c|c|}
\hline Distribution & Lindley & APTL \\
\hline \multirow{2}{*}{ Parameter estimates } & $\theta=0.86826238$ & $\hat{\alpha}=129.772309$ \\
\cline { 2 - 3 } & & $\theta=1.649062$ \\
\hline Kolmogorov-Smirnov & 0,246674 & 0,094189414 \\
\hline
\end{tabular}

Figure 3 provide the comparison by plot of pdf Lindley and APTL distributions and table 2 provide information about estimated parameters and Kolmogorov-Smirnov statistics for the data set. In this case, from Figure 3, it can be seen that APTL distribution fits well to the data set and the Kolmogorov-Smirnov test we reject the hypothesis that the Lindley distribution does not suitable to modeled the data and we do not reject the hypothesis that the data come from APTL distribution considered with a significance level at $\alpha=0.05$.

\section{Conclusion}

In this paper, we proposed a new distribution with two parameters, so-called the APTL distribution. The proposed APTL distribution has two shape parameters. This new APTL distribution is appropriate in modelling data with decreasing or unimodal shaped of probability density function, and increasing, decreasing, and upside-down bathtub shaped hazard rates. APTL distribution provides a better fit than Lindley distribution for modelling data. We hope that the APTL distribution can be used on wider sets of applications.

\section{References}

Efron, B. (1988). Logistic regression, survival analysis, and the Kaplan-Meier curve. 
Journal of the American statistical Association, 83(402), 414-425.

Ghitany, M. E. (2008). Lindley distribution and its application. Mathematics and computers in simulation, 78(4), 493-506.

Kuş, C. (2007). A new lifetime distribution. Computational Statistics \& Data Analysis, 51(9), 4497-4509.

Lindley, D. V. (1958). Fiducial distributions and Bayes'theorem. Journal of the Royal Statistical Society. Series B (Methodological), 78(4), 102-107.

Mahdavi, A. \&. (2017). A new method for generating distributions with an application to exponential distribution. Communications in Statistics-Theory and Methods, 6543- 6557. 